\title{
XENON1T: the start of a new era in the search for Dark Matter
}

\author{
Sara Diglio* (on behalf of the XENON collaboration) \\ Subatech, École des Mines de Nantes, CNRS / IN2P3, Université de Nantes, Nantes, France \\ E-mail: diglio@subatech.in2p3.fr
}

\begin{abstract}
The understanding of the nature of the mysterious Dark Matter in our Universe represents one of the biggest challenges in frontier science today. There are strong evidences for its existence from astrophysics and cosmological measurements. A number of proposed candidates have been put forward over time: one of the most compelling are Weakly Interacting Massive Particles (WIMPs). The XENON dark matter project aims at finding direct evidence for the scattering of WIMPs with target nuclei in an ultra-low background dual-phase xenon Time Projection Chamber (TPC) based detector. After the successful operation of the XENON100 instrument the next generation XENON1T is ready to start data taking at the italian Gran Sasso underground INFN National Laboratory.

In this proceeding an introduction to the direct dark matter detection methods will be reviewed; the latest results of the XENON100 experiment will be firstly revised, then the special challenges related to a ton-scale liquid xenon detector will be discussed and the current status of the XENON1T experiment as well as its prospects and projected physics reach will be presented.
\end{abstract}

11th International Workshop Dark Side of the Universe 2015

14-18 December 2015

Yukawa Institute for Theoretical Physics, Kyoto University Japan

\footnotetext{
*Speaker.

${ }^{\dagger}$ We gratefully acknowledge support from Region des Pays de la Loire
} 


\section{Introduction}

In the past decade, breakthroughs in cosmology have transformed our understanding of the Universe. A wide variety of observations now support a unified picture in which the visible stars and gas in all galaxies, including our own, are immersed in a much larger cloud of non-luminous matter which outweighs the luminous component by at least an order of magnitude. Evidence for the existence of Dark Matter (DM) in the Universe has steadily accrued since the 1930s, when the astronomer Fritz Zwicky found that additional matter, beyond that which is luminous, was needed to explain gravitational binding of clusters of galaxies. In particular, rotation curves of galaxies usually exhibit a characteristic flattening out at large distances towards and beyond the edge of the visible galaxy disk, implying the existence of a DM halo. Since then, evidence from galactic rotation curves, gravitational lensing, hot gas in galactic clusters, precision measurements of the Cosmic Microwave Background and measurements of large scale structure in the Universe all support the existence of DM. By combining these data sets with many others - including the density of baryons as deduced from Big Bang nucleosynthesis and various measurements of Dark Energy in the Universe - current estimates put the DM fraction of mass-energy density of the observable Universe at $\sim 26 \%$, baryonic matter makes up $\sim 5 \%$ while the remainder is accounted for by dark energy [1]. The existence of DM is at present one of the strongest pieces of evidence that the current theory of fundamental particles and forces, summarized by the Standard Model (SM) of particle physics, is incomplete. The nature of this non-baryonic component is still unknown, and the resolution of the "Dark Matter puzzle" is of fundamental importance to cosmology, astrophysics, and elementary particle physics. A number of proposed candidates have been put forward over time: one possibility motivated by other considerations in elementary particle physics is that DM consists of undiscovered elementary particles that arise naturally in many theories beyond the SM. One of the most compelling candidates are Weakly Interacting Massive Particles (WIMPs). These particles are well motivated, not only because they resolve the DM puzzle, but also because they simultaneously solve longstanding problems associated with the SM of particle physics. WIMPs appear naturally in many model frameworks designed to understand the weak force, including supersymmetric theories, theories with extra spatial dimensions, and others. These particles are predicted to be formed in the early universe and subsequently gravitationally clustered in association with baryonic matter. WIMPs are expected to interact weakly with normal matter and have mass near the weak scale $(100 \mathrm{GeV}-1 \mathrm{TeV})$.

There are several complementary methods used to determine the particle nature and properties of DM. Direct detection experiments examine the interaction of WIMP particles with a detector as they elastically scatter off nuclei in the target. DM may also be discovered indirectly by finding evidence of WIMP pairs annihilating somewhere in the galactic neighborhood. These indirect signals are important targets for a vast array of experiments, including neutrino telescopes, spacebased anti-matter searches and $\gamma$-ray telescopes. Finally, evidence for WIMPs may also come from collider searches. The Large Hadron Collider experiments are already exploring the weak scale as they have excellent ability for seeing the missing energy signals characteristic of WIMP that could either be produced directly or via decays of other new matter states. 
In the following sections the DM direct detection principle will be described, with a particular focus on the XENON project.

\section{Dark Matter direct detection}

Large efforts have been pursued to develop experiments which are able to directly test the particle nature of DM. Given the low interaction strength expected for the DM particle, the probability of multiple collisions within a detector is negligible, thus the signature results in a recoil spectrum of single scattering events. In the most common approach, the experiments attempt to measure the nuclear recoils energy produced by collisions between DM candidates and detectors target nuclei. Another possible DM signature is represented by the so-called "annual modulation". As consequence of the Earth rotation around the Sun, the speed of the DM particles in the Milky Way halo relative to the Earth is largest around June 2nd and smallest in December. Moreover, the possibility to measure the direction of the recoils would improve the discriminating power of the experiments, since a strong angular dependence of WIMP interactions is expected [2].

In order to maximize the possibility to observe a DM signal, it is requested that the detectors meet the following conditions:

- ultra-low background experimental environment;

- large detector mass to enhance the interaction probability inside the target;

- low energy threshold to detect the smallest recoil energies;

- good discrimination power against particle interactions that might mimic WIMP collisions;

- stable detector performance over time scales of a few years.

Different technologies have been explored so far to fulfill the above requirements, a detailed summary can be found in [3]. An experimental technique which demonstrated to be very effective for the direct DM detection consists of a target made of liquefied noble gases, as it will be discussed in the following.

\subsection{Noble gas detectors}

As detector media, noble gases present interesting advantages such as the possibility to create homogeneous detection volumes at a reasonable cost with high particle stopping powers. Another advantage of liquid noble gas detectors is the accurate interaction position reconstruction thanks to fast scintillation response and ionisation signal in presence of a drift field, and the low transverse diffusion for drifting electrons. With adequate position resolution the detectors can employ selfshielding to avoid the surface contamination problems that plague solid state detectors. This allows the exploitation of a good fiducialisation in order to reduce background exposition for final search within the active volume of the detector.

Noble gas detectors are relatively simple, and the liquids can be purified in situ. The higher operating temperatures of noble liquids allow more straightforward and less expensive cryogenic 
systems with respect to other technologies. With their large detector mass the interaction probability inside the target is increased, allowing for a better sensitivity at large WIMP masses (above $\sim 10 \mathrm{GeV} / \mathrm{c}^{2}$ ). These detectors usually use either Argon (Ar) or Xenon (Xe), and eventually Neon $(\mathrm{Ne})$, in liquid phase (LAr or LXe, eventually LNe) as target material. They can be either single phase (XMASS, DEAP, CLEAN) or dual-phases (WARP, XENON, DarkSide, LUX, ArDM), in this latter case, a Time Projection Chamber (TPC) with liquid and gaseous phase of the same material will be used. The first category exploit the scintillation light in the liquid, possibly using pulse shape discrimination to select nuclear recoils. Dual-phases detectors may combine the above techniques with the ionization charge measurements resulting in a more efficient discrimination between nuclear recoils and electronic recoils ( $\gamma$-rays, betas), which is an important method to reduce the background of the experiment as it will be discussed in section 3.1.

In the following sections the focus will be on the XENON project: the functioning of the XENON dual-phase TPC will be described in sec 3.1, recent results from XENON100 will be discussed in 3.2 and an overview of status of the XENON1T detector including design characteristics and its scientific reach will be presented in 3.3.

\section{The XENON project}

The XENON Collaboration is working on the search for DM and has chosen the dual-phase (Liquid-Gas) Xe TPC as detecting medium.

At present, the XENON Collaboration consists of 21 institutions and about 130 collaborators from all over the world. The XENON detectors have been operating at the Laboratori Nazionali del Gran Sasso (LNGS) in Italy at an average depth of $3600 \mathrm{~m}$ water equivalent since 2006. The XENON Collaboration completed first the XENON10 detector [4, 5], consisting of $25 \mathrm{~kg}$ of LXe, then the XENON100 detector [6, 7], consisting of $161 \mathrm{~kg}$ of LXe. After the successful operation of the XENON100 experiment - for many years the most sensitive WIMP detector in the world the next generation XENON1T [8,9] has been inaugurated in November 2015. First science data are expected by end of spring 2016. With total Xe mass of $\sim 3.5$ ton, XENON1T is the first multiton LXe experiment for DM searches world-wide. It has a design sensitivity for spin-independent WIMP-nucleon cross section close to $10^{-47} \mathrm{~cm}^{2}$ for a WIMP mass of $\sim 50 \mathrm{GeV} / \mathrm{c}^{2}$. This is about a factor 100 below the XENON100 best limit and will be reached with an exposure of 2 ton per year [9]. To achieve another order of magnitude in sensitivity, the XENON Collaboration plan to build and install in the same vacuum cryostat a new detector, XENONnT, with more than twice the LXe mass and with even lower background. The new experiment will use the same shield, cryogenic plants and data acquisition system of XENON1T, making rapid realization. XENONnT is set to start operating by 2019 .

Table 1 reported characteristics and expectations of the different XENON experiments. 


\begin{tabular}{|c|c|c|c|}
\hline & XENON10 & XENON100 & XENON1T \\
\hline Total Xe mass (kg) & 25 & 161 & 3500 \\
\hline Target Xe mass (kg) & 14 & 62 & 2000 \\
\hline Fiducial Xe mass (kg) & 5.4 & 48 & 1000 \\
\hline $\begin{array}{l}\text { TPC size }(\mathrm{cm}): \\
\text { diameter, height }\end{array}$ & $\mathrm{D}=25, \mathrm{H}=15$ & $\mathrm{D}=30, \mathrm{H}=30$ & $\mathrm{D}=96, \mathrm{H}=95$ \\
\hline Photo sensors & $\begin{array}{l}891 " \text { PMTs } \\
\text { (R8520) }\end{array}$ & $\begin{array}{l}242 \text { 1" PMTs } \\
\text { (R8520) }\end{array}$ & $\begin{array}{l}248 \text { 3" PMTs } \\
\text { (R11410) }\end{array}$ \\
\hline${ }^{n a t} \mathrm{Kr} / \mathrm{Xe}(\mathrm{ppt})$ & 5000 & 20 & 0.2 \\
\hline $\begin{array}{l}{ }^{222} \mathrm{Rn} / \mathrm{Xe} \\
(\mu \mathrm{Bq} / \mathrm{kg})\end{array}$ & 60 & 65 & 10 \\
\hline $\begin{array}{l}\text { ER bkg in FV } \\
\text { (evts / keV·kg.day) }\end{array}$ & $\sim 1$ & $\sim 5.3 \times 10^{-3}$ & $\sim 1.8 \times 10^{-4}$ \\
\hline $\begin{array}{l}\text { WIMP-nucleon } \\
\text { cross section limits }\end{array}$ & $\begin{array}{l}4.5 \times 10^{-44} \mathrm{~cm}^{2} \\
@ 30 \mathrm{GeV} / \mathrm{c}^{2} \\
\text { in } 58.6 \text { live days } \times 5.4 \mathrm{~kg}[4]\end{array}$ & $\begin{array}{l}2.0 \times 10^{-45} \mathrm{~cm}^{2} \\
@ 55 \mathrm{GeV} / \mathrm{c}^{2} \\
\text { in } 224.6 \text { live days } \times 34 \mathrm{~kg}[7]\end{array}$ & $\begin{array}{l}\sim 1.6 \times 10^{-47} \mathrm{~cm}^{2} \\
@ 50 \mathrm{GeV} / \mathrm{c}^{2} \\
\text { in } 2 \text { years } \times 1 \mathrm{t}[9]\end{array}$ \\
\hline
\end{tabular}

Table 1: Comparison of characteristics among the XENON experiments. Measurements are reported for XENON10 and XENON100 while expectations are shown for XENON1T.

\subsection{The XENON detectors working principles}

The XENON detectors consist in Time Projection Chambers (TPCs) filled with Xe in liquid (LXe) and gaseous (GXe) phase.

The detection principle is presented in Fig.1. An interaction in the liquid Xe target generates prompt scintillation light and ionization electrons. Electrons are drifted by an electric field toward the gaseous phase and there detected through a process of proportional scintillation. Both primary and secondary light are detected by photomultiplier tubes (PMTs) placed on the top and on the bottom of the cylinder. The interaction point is reconstructed in three dimensions, with the $\mathrm{x}, \mathrm{y}$ coordinates determined from the hit pattern of the localised signal on the top PMTs array, while the $\mathrm{z}$ coordinate is deduced by means of the drift time between the two light signals. The scintillation signal in the gas phase, called $\mathrm{S} 2$, is delayed relative to the $\mathrm{S} 1$ (prompt scintillation) signal by the drift time of the electrons through the liquid. Recoil ions produce a dense region of ionization whereas electrons produce a diffuse track. Due to their different ionization densities, there is more recombination of electron-ion pairs in nuclear recoils compared to recoiling electron. Consequently, compared to those electrons, nuclear recoils produce prompt scintillation light but have fewer un-recombined ionization electrons. Discrimination between background due to $\gamma$ and $\beta$ interactions (electronic recoils, ER) and nuclear recoils (WIMP signal and neutron background, $\mathrm{NR}$ ) is therefore possible by measuring the ratio of prompt scintillation light signal to collected ionization charge signal. The ratio S2/S1 is about 5 times bigger for electron events compared to 


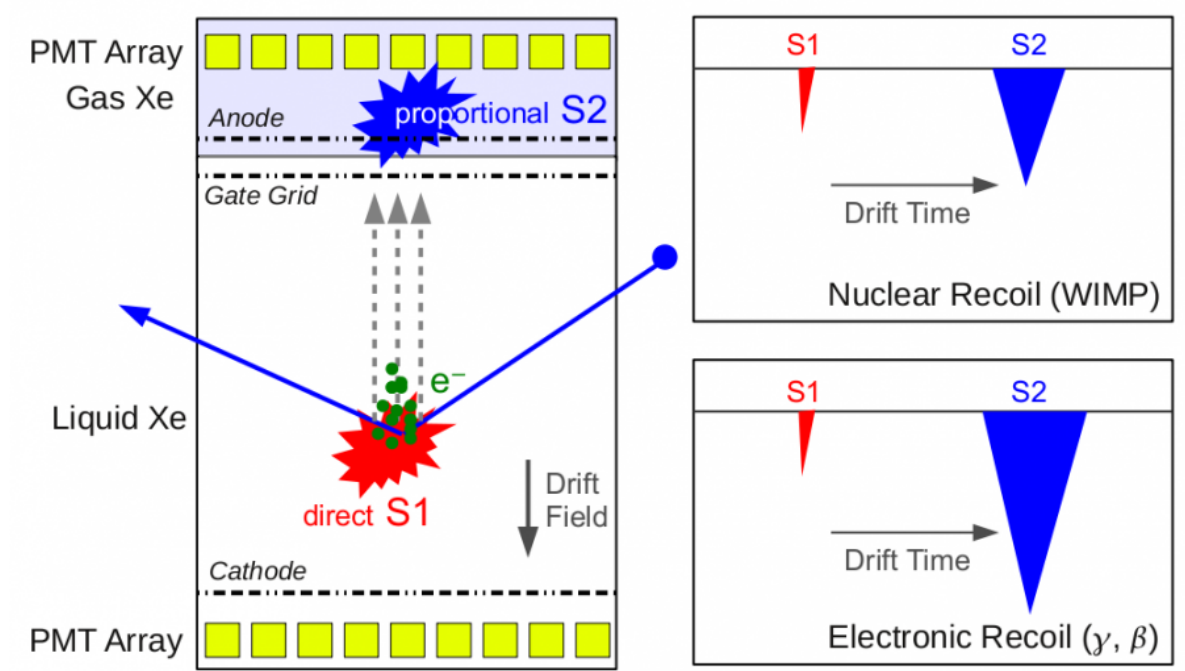

Figure 1: Left: working principle of the XENON liquid/gas dual-phase TPC. Right: sketch of the waveforms of nuclear recoils (WIMPs and neutrons, NR) and electronic recoils ( $\gamma$ and $\beta$ background, ER), showing the different ratio of the charge (S2) and light (S1) signals for the two types of events.

nuclear events. This method allows a good discrimination of signal from background.

Among the advantages of using $\mathrm{Xe}$ as a target detector, there are the two following: it is the noble gas with the best stopping power; it has almost no intrinsic radioactivity, since all isotopes of Xe found in nature are stable, or long-life double-beta emitter ( ${ }^{136} \mathrm{Xe}$, Half-life: $\tau \sim 10^{21} \mathrm{yr}$ ). This property is crucial for low background experiments. Furthermore, due to the much higher atomic mass with respect to other noble gases, Xe has a higher expected signal rate. In fact, the spin-independent WIMP-nucleus interaction rate is proportional to the square of the nuclear mass, and so the WIMP signal should be greatly enhanced for large nuclei. In addition LXe has the advantage of containing almost $50 \%$ of non zero spin isotopes, ${ }^{129} \mathrm{Xe}$ and ${ }^{131} \mathrm{Xe}$, providing additional sensitivity to spin-dependent WIMP interactions. Finally, Xe liquefies at temperatures below 165 $\mathrm{K}$ at 1 bar, therefore the cryogenic system needed to keep it at such temperature is relatively easier than for lighter noble gas that will request lower temperatures for their cryogenic.

XENON detectors rely on careful selection of low-background materials to suppress internal sources of background, self-shielding against $\gamma$ rays from external sources and good event position resolution to define fiducial volume (FV). External shielding and an active veto are also required to reject $\beta, \gamma$ and neutrons. In addition, the high atomic number and the large density make the inner volume of the Xe target virtually free of electromagnetic backgrounds.

More details about detector components and background predictions will be given in section 3.3.

\subsection{Results from XENON100}

The XENON100 detector is operative since 2008. In 2012 it has reached the sensitivity of $2.0 \times 10^{-45} \mathrm{~cm}^{2}$ at $55 \mathrm{GeV} / \mathrm{c}^{2}$ and $90 \%$ confidence level on spin-independent elastic WIMP- 
nucleon scattering cross section [7] (fig. 2 on the left). The result for spin-dependent interactions on neutrons is reported on the right hand side of fig. 2 [10]. These limits result from the analysis of data taken over an integrated period of 225 live-days.
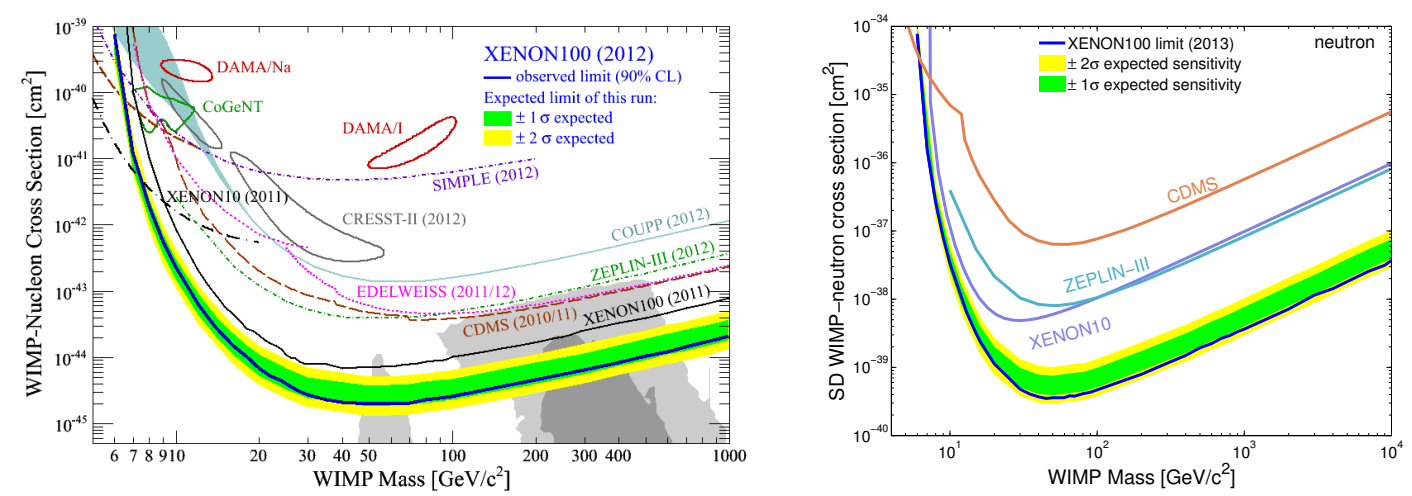

Figure 2: Left side: Spin-Independent (SI) WIMP-nucleon cross section limits [7]. Right side: SpinDependent (SD) WIMP-neutron cross section limits [10]. Green (yellow) bands on both figures are the $1 \sigma(2 \sigma)$ uncertainty on the expected sensitivity. The resulting exclusion limits (90\% CL) in blue are also represented. Other experimental results are also shown for comparison.

The same XENON100 data have been used to investigate the axions and axion like particles (ALPs) hypotheses [11] and to perform analysis aiming at reconciling the apparent tension between XENON100 and other experiments with the possible DM signal observed by the DAMA/LIBRA collaboration [12]. The former search is well motivated by models describing axions and ALPs as cold dark matter candidates which interact predominantly with atomic electrons: ER events have been analyzed and upper limits have been set. Figure 3 on the left shows that for solar axions with masses below $1 \mathrm{keV} / \mathrm{c}^{2}$, values of the axion-electric coupling constant $g_{A e}$ larger than $7.7 \times 10^{-12}$ are excluded (90\% C.L.). Figure 3 on the right shows the upper limit on ALPs-electron coupling in the $5-10 \mathrm{keV} / \mathrm{c}^{2}$ mass range.
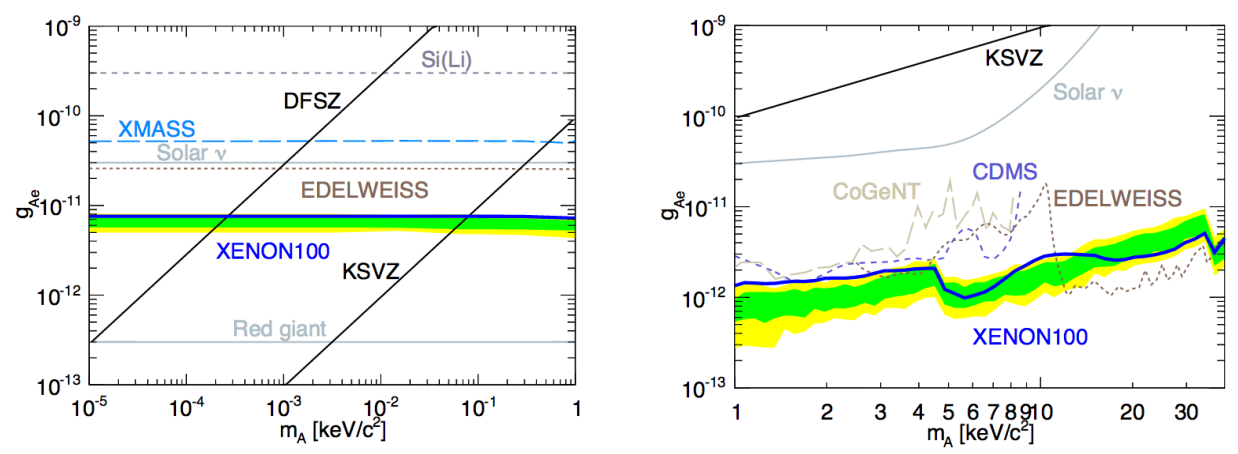

Figure 3: Left side: the XENON100 limits on solar axions (blue line) at 90\% C.L. Right side: the XENON100 limit (90\% CL) on ALP coupling to electrons as a function of the mass, under the assumption that ALPs constitute all the dark matter in our galaxy (blue line). The expected sensitivities at $1 \sigma$ (green) and $2 \sigma$ (yellow) are shown in both plots. Limits form other experiments are also shown for comparison [11]. 
Concerning the possible DM signal observed by the DAMA/LIBRA, since the detector has no discrimination power between NR and ER, a possibility to reconcile the observed signal with the null-results from other experiments, would consist in assuming leptophilic DM. In order to test such hypothesis, the XENON Collaboration performed two analyses both focused on the interaction between a DM candidate and electrons in the detector medium. The first study used the overall ER rate and allowed to exclude three leptophilic models as explanation for the DAMA/LIBRA signal [13]. A summary of the excluded models is reported in table 2.

\begin{tabular}{lc} 
Excluded models & Confidence level \\
\hline \hline WIMP axial-vector coupling to electrons & $4.4 \sigma$ \\
\hline Kinematically mixed mirror DM & $3.6 \sigma$ \\
\hline Luminous DM & $4.6 \sigma$ \\
\hline
\end{tabular}

Table 2: Exclusion limits on different leptophilic models that could have explained the DAMA/LIBRA signal [13].

Fig. 4 on the left shows the DAMA/LIBRA modulated energy spectra, interpreted as WIMPs scattering through axial-vector interactions for the models listed in table 2, as it would be seen by the XENON100 experiment.
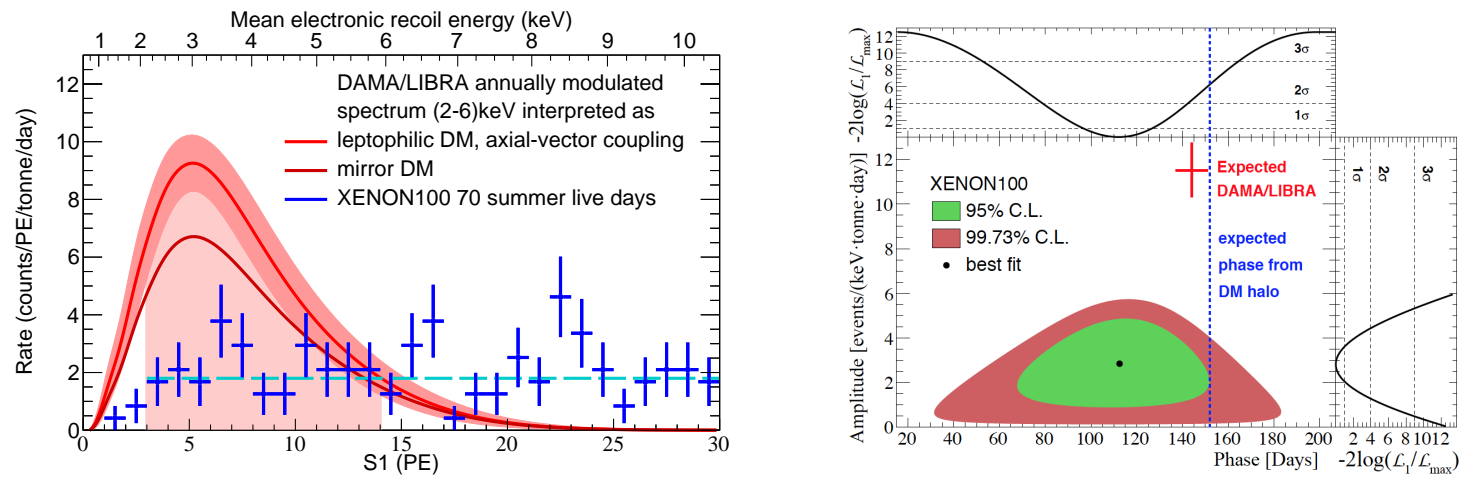

Figure 4: Left side: The DAMA/LIBRA modulated energy spectra for different models (red and dark red), interpreted as WIMPs scattering through axial-vector interactions, as it would be seen in the XENON100 detector. The $1 \sigma$ band includes statistical and systematic uncertainties. The (blue) data points are XENON100 data from the 70 summer live days with their statistical uncertainty. The expected average XENON100 rate is also shown (dashed cyan) [13]. Right side: The XENON100 best-fit, 95\% and 99.73\% confidence level contours as a function of modulation amplitude and phase relative to January 1, 2011 for the period of 1 year [14].

The other study exploited the periodic variations of the electronic recoil event rate in the (2-6) $\mathrm{keV}$ energy range [14]. The measured phase of $112 \pm 15$ days disfavored the interpretation of a modulation due to standard DM halo at $2.5 \sigma$ confidence level, as it can be seen in fig. 4 on the right. In addition, the annual modulation interpreted as a DM signature with axial-vector coupling of WIMPs to electrons was also excluded at $4.8 \sigma$ confidence level. 


\subsection{Design and status of the XENON1T}

As part of the evolution of the XENON program, in the same underground laboratory where XENON100 is located, the commissioning of XENON1T is ongoing: science data-taking is projected to start by end of spring 2016. The construction of XENON1T started in 2013. Figure 5 on the left shows a picture of the XENON1T experimental area in the Hall B of the LNGS. A drawing of the TPC and its components is represented on the right of figure 5.
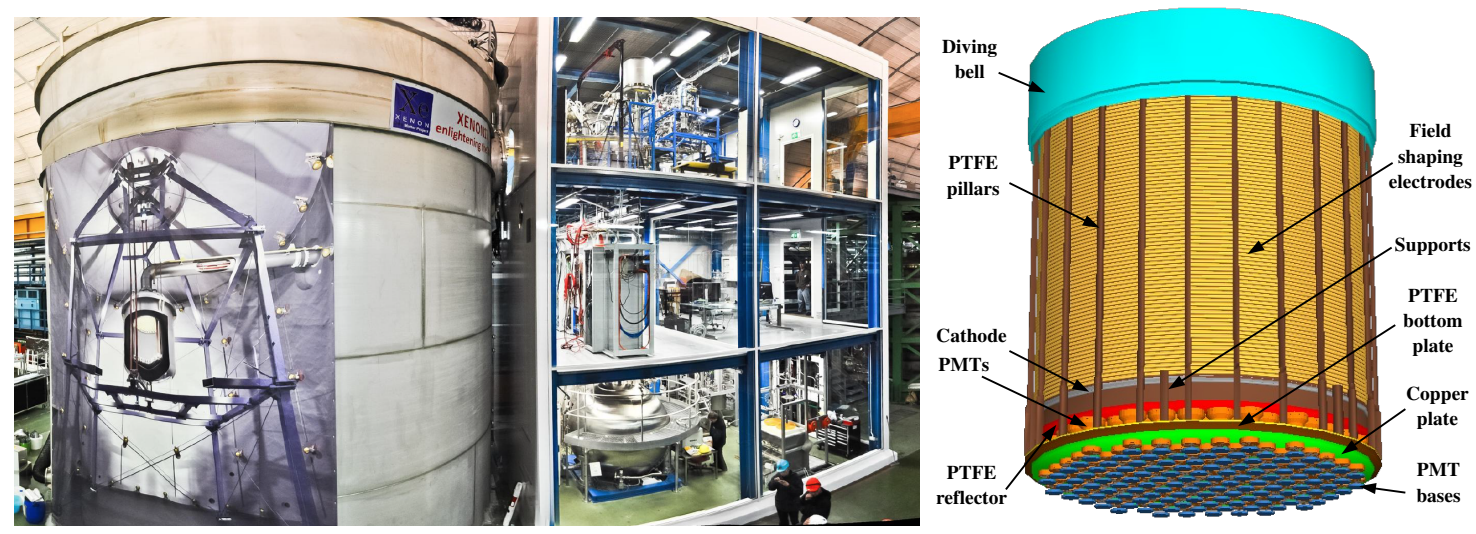

Figure 5: Left side: XENON1T experimental area in the Hall B of the LNGS. Right side: drawing of the TPC and its components [9].

The XENON1T experiment has been designed to increase the sensitivity of XENON100 by about two orders of magnitude. To do this, a larger unprecedented target mass and lower background levels with respect to previous experiments are required. A special care has been taken by the XENON Collaboration in order to fulfill these requirements. In particular, to handle the total mass of more than three tons of $\mathrm{Xe}$, a special storage/recovery system, cryogenic infrastructures and purification systems were firstly designed and then built. In addition, in order to reach very low intrinsic radioactivity, all detector materials close to the target volume have been thoroughly screened and where chosen after a screening campaign, a water Cherenkov muon veto and a cryogenic distillation column were constructed. A Monte Carlo simulation based on GEANT4 has been developed to estimate the detector sensitivity to the interaction between a DM candidate and the detector medium [9]. In the following sections the different subsystems constituting the XENON1T detector will be introduced and the experiment scientific reach will be presented.

\subsubsection{XENON1T subsystems}

\section{Liquid Xenon Detector}

The dual-phase TPC has the longest drift and largest active mass of LXe of any TPC built to-date. It consists of a cylinder of $95 \mathrm{~cm}$ height and $96 \mathrm{~cm}$ diameter filled with active LXe target of about 2 tons and instrumented with 248 3"-inch Hamamatsu PMTs (R11410) for the simultaneous detection of scintillation light and ionization charge via proportional gas scintillation. The materials used for PMTs have been carefully selected in order to keep the level of their radioactivity as low as possible [15]. The details of the PMT performance are described in [16, 17, 18, 19]. Their 
average quantum efficiency is of $35 \%$ at Xe scintillation of $178 \mathrm{~nm}$ and their single photoelectron resolution is of about $30 \%$. An homogenous electric drift field of $1 \mathrm{kV} / \mathrm{cm}$ will be supplied inside the active volume by a cathode at bottom and a gate at a few $\mathrm{mm}$ below the liquid level. A sketch of the external part of the TPC including its components is presented in fig. 5 on the right.

\section{Water Cherenkov Muon Veto}

A water tank $10.5 \mathrm{~m}$ high and $9.6 \mathrm{~m}$ in diameter, filled with $700 \mathrm{~m}^{3}$ of deminaralized water, will act as an active shield around the LXe detector against low energy $\gamma_{\mathrm{s}}$ and neutrons. The water system is equipped with 84 8-inch high quantum efficiency PMTs sensitive to Cherenkov light aiming at identifying cosmic ray muons that may induce high energy neutrons that can reach the TPC and mimic WIMPs interactions, resulting in source of background for XENON1T. This system will thus operate as a Water Cherenkov Muon Veto with an estimated efficiency in tagging muon induced neutrons larger than $99.7 \%$ in case also the parent muon is hitting the water tank (this happens in one third of the cases) and larger than $71.4 \%$ otherwise. The muon induced neutron background in 1 ton of fiducial volume is estimated to be less than 0.01 per year [20].

The system will also provide access points and feedthroughs for water purification, calibration sources and detector leveling.

\section{Cryostat}

An ultra vacuum, thermally insulated system made of low-radioactivity material has been conceived to host the LXe detector at a temperature of $-95{ }^{\circ} \mathrm{C}$ and a pressure of 2 bar. The system consists of two independent cylindrical vessels: an inner vessel $1.5 \mathrm{~m}$ high and $1.1 \mathrm{~m}$ of diameter (insulated with multi-layer insulators and vacuum) and an outer vessel made to accommodate a larger inner vessel for a more massive future detector (XENONnT). A $7.5 \mathrm{~m}$ long line, doublewalled and vacuum insulated, carries independent inner pipes for PMT signals/high voltage cables and for Xe filling/recovery. An additional separate line carries the high voltage (whose maximum value is of $150 \mathrm{kV}$ ) to the detector through a custom-designed ultra high vacuum fiber feedthrough. The system has been optimized via finite element method (FEM) stress analysis to simultaneously minimize mass contributing to background while maximizing allowable working pressure.

\section{Cryogenic and Purification Systems}

The cryogenic system has been designed to liquefy $3500 \mathrm{Kg}$ of $\mathrm{Xe}$ and maintain it in liquid form inside the cryostat at constant pressure and cold temperature for long term operations. The system consists of two redundant pulse tube refrigerator (PTR) towers: one PTR whose cooling power is of about $200 \mathrm{~W}$ at LXe temperature, and a second LN2 cooling system to be used in case of emergency like long term power-outage [21]. In the same service building where the cryogenic system is located, a purification system consisting of commercial high-flow hot getters has been installed to clean the Xe from electronegative impurities via continuous circulation of gas trough heated getters.

\section{Storage and Recovery}

The Storage and Recovery system (ReStoX) is an self-made cryogenic vessel designed to store, purify and recover up to 7.6 tons of Xe both in liquid or in gaseous phase under high purity conditions. Before transferring the Xe into ReStoX, it is stored in bottles: a gas handling and impurity control 
measures impurities level of each cylinder of Xe gas using a dedicated Gas Chromatograph. This system connect and analyze up to four gas cylinders to recuperate gas residuals during detector filling. Due to the large scale of the XENON1T detector, the ReStoX component is very strategic to improve the performance of the experiment: it is able to store and purify liquid Xe in advance, so to dramatically shorten the purification dead time when the detector will be filled. Similarly, it can also recover it in case of temporary problems, preventing losses of Xe. ReStoX consists of a double walled, high pressure ( 72 bar) vacuum insulated sphere of 2.1 meter diameter, cooled by LN2 and by an internal LN-based condenser.

\section{Cryogenic Distillation Column}

An active removal of ${ }^{85} \mathrm{Kr} \beta$-emitter contamination from the $\mathrm{Xe}$ is realized trough the cryogenic distillation column [22]. XENON1T requires $\mathrm{Kr}$ concentration less than 0.2 parts-per-trillion (ppt) while commercially available Xe contains $\mathrm{Kr}$ of about 5 parts-per-billion (ppb). The column uses the difference in vapour pressures between $\mathrm{Kr}$ and $\mathrm{Xe}$ to remove $\mathrm{Kr}$ from $\mathrm{Xe}$. By using measurement systems with sub-ppt precision [23, 24], the functionality of the column has been verified.

\section{Data acquisition and computing}

An high speed data acquisition system (DAQ) has been developed in order to read the data from PMTs, select interesting events, store data to file, process raw data. It has been designed to reach the lowest possible threshold and to select online data using individual channel readout and online trigger on PC farm. The maximum DAQ rate is expected to be around $1000 \mathrm{~Hz}$ for calibration data taking, while the DM trigger is estimated to reach $2 \mathrm{~Hz}$. In order to handle the total amount of data, computing/analysis facilities have been put in place: they consist in both local and remote systems. Concerning the latter, a consistent use of sharing resources (GRID) is foreseen, in particular to process and store raw data and Monte Carlo simulations.

\subsubsection{Monte Carlo simulation}

A Monte Carlo (MC) simulation fully based on GEANT4 has been developed in order to reproduce via software the performance of the XENON1T detector, and predict the sensitivity of the experiment. Inputs from screening campaign from all detector components have been used to tune the MC. The simulation has been used to estimate the rate of the different sources of ER and NR backgrounds which will affect the discovery potential of XENON1T.

Radioactivity from the different detector components will result in ER backgrounds within the detector FV, whose main contribution ( $\sim 85 \%$ of the total ER background) comes from the decay of the ${ }^{222} \mathrm{Rn}$ daughters. Other sources of ER background are: the radioactive isotope ${ }^{85} \mathrm{Kr}$ that is contained in the ${ }^{n a t} \mathrm{Kr}$ from which the Xe is extracted ( $\sim 4 \%$ of the total ER background), solar neutrinos that scatter elastically off the electrons of the medium ( $\sim 5 \%$ of the total ER background) and ${ }^{136} \mathrm{Xe}$ (contained in the natural $\mathrm{Xe}$ ) that is responsible for double-beta decays and that contributes to about $1 \%$ of the total ER background.

Figure 6 on the left shows the spatial distribution of the ER background events from the detector materials inside the whole active volume, in the energy range between 1 and $12 \mathrm{keV}$. FVs corresponding to different target masses are also indicated. 
Neutrons can produce NRs via elastic scattering off Xe nuclei, mimicking the WIMP-nucleon scattering. Among the main contributors to the NR backgrounds we can list: radiogenic neutrons from detector components, neutrons that are produced by the interaction of cosmic muons with the rock and concrete around the underground laboratory and with the detector materials (so called muoninduced neutrons) and finally NRs resulting from the coherent neutrino-nucleus scattering (CNNS). Figure 6 on the right shows the energy spectrum of each contributor of the total NR background listed above, in 1 ton FV.
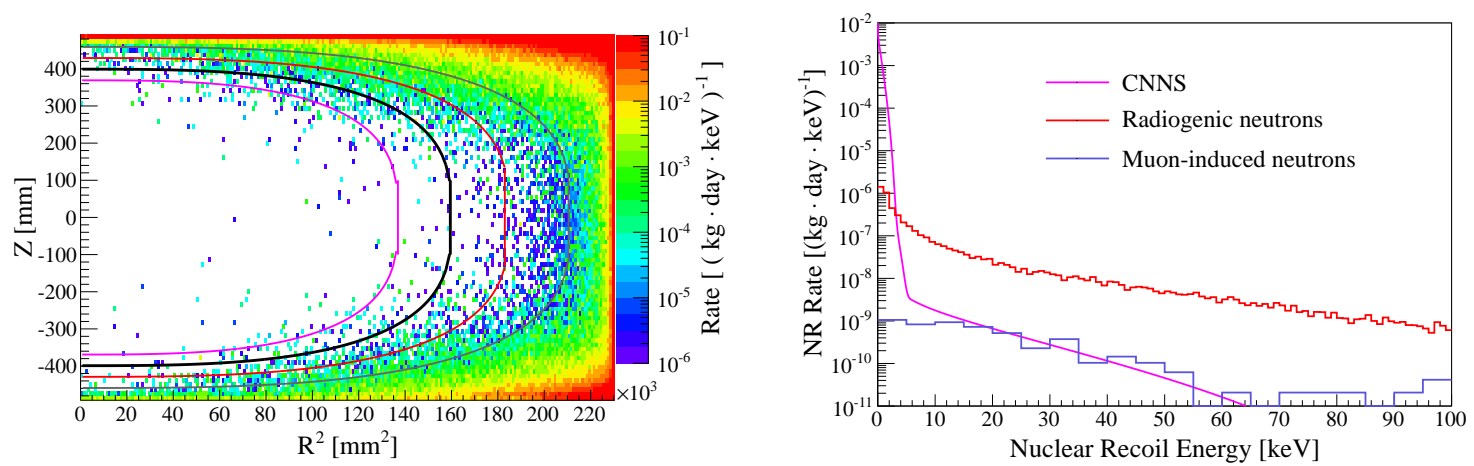

Figure 6: Left side: Spatial distribution of the ER background events from the detector materials inside the active LXe volume, in the 1-12 keV energy range. The thick black line indicates the reference 1 ton FV. Additional FVs corresponding to $800 \mathrm{~kg}, 1250 \mathrm{~kg}$ and $1530 \mathrm{~kg}$ are also indicated by the purple, red and brown lines, respectively. The white regions present a background rate smaller than $10^{-6}(\mathrm{~kg} \cdot \text { day } \cdot \mathrm{keV})^{-1}$. Right side: Energy spectrum of the different contributions to the NR background events in 1 ton FV. Both figures are from [9].

The separate contributions of ERs and NRs together with the total background spectrum as a function of $\mathrm{S} 1$ is represented in figure 7. Events with $\mathrm{S} 2>150 \mathrm{PE}$ are selected assuming a $99.75 \%$ ER rejection with a flat $40 \%$ NR acceptance. Event rates for three examples of WIMP signals corresponding to three different masses and expected cross sections are also shown.

In table 3 the predicted backgrounds in XENON1T assuming 99.75\% ER discrimination with a flat $40 \%$ NR acceptance are summarized. The reported numbers have been evaluated in 1 ton $\mathrm{FV}$ and in the S1 range between 3 and $70 \mathrm{PE}$ (on average, it corresponds to the NR energy range between 4 and $50 \mathrm{keV}$ ) considering a 2 years exposure.

\begin{tabular}{lc} 
Background Sources & Events \\
\hline \hline $\begin{array}{l}\text { Electron Recoils : } \\
\text { materials }+{ }^{222} \mathrm{Rn}+{ }^{85} \mathrm{Kr}+{ }^{136} \mathrm{Xe}+\text { Solar neutrinos }\end{array}$ & 3.25 \\
\hline $\begin{array}{l}\text { Nuclear Recoils : } \\
\text { radiogenic neutrons }+ \text { muon-induced neutrons + CNNS }\end{array}$ & 0.91 \\
\hline
\end{tabular}

Table 3: Expectation values of events in XENON1T in 2 years exposure, within the 1 ton FV and in the S1 range between 3 and $70 \mathrm{PE}$ assuming 99.75\% ER discrimination with a flat 40\% NR acceptance [9]. 


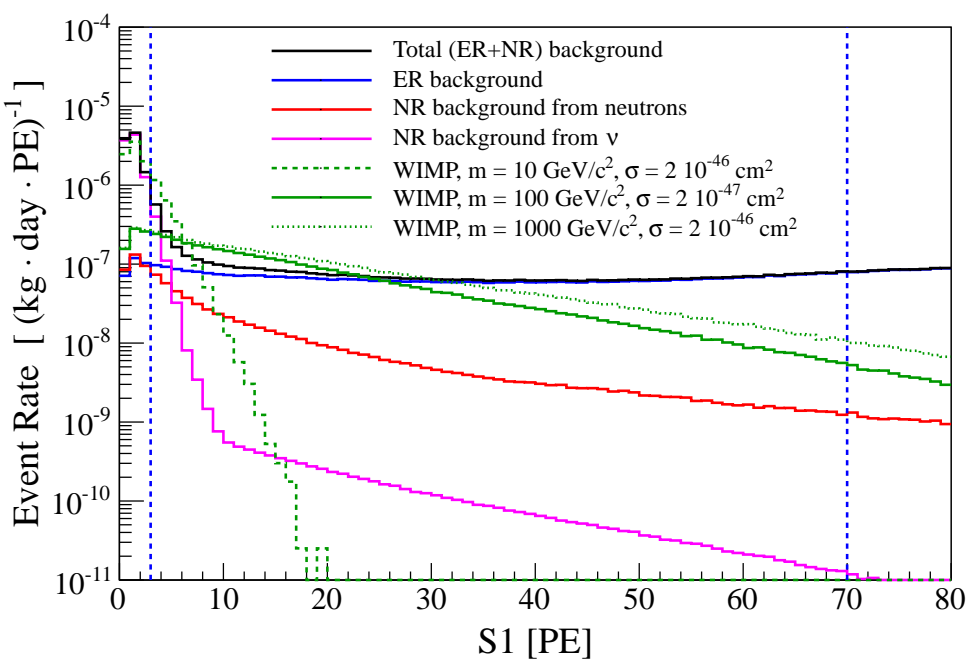

Figure 7: Spectrum of the total background and of its components as a function of S1. Event rates for three examples of WIMP signals corresponding to three different masses and expected cross sections are also shown. The vertical dashed blue lines delimit the S1 region used in the sensitivity calculation. Events with S2 > $150 \mathrm{PE}$ are selected assuming a $99.75 \%$ ER rejection with a flat 40\% NR acceptance [9].

Figure 8 shows the sensitivity expected by the XENON1T experiment to spin-independent WIMP-nucleon interaction after 2 years of exposure in 1 ton FV. The minimum cross section of $1.6 \times 10^{-47} \mathrm{~cm}^{2}$ is achieved for a mass $m_{\chi}=50 \mathrm{GeV} / \mathrm{c}^{2}$. For comparison results from other experiments as well as prediction for XENONnT are shown in the same figure.

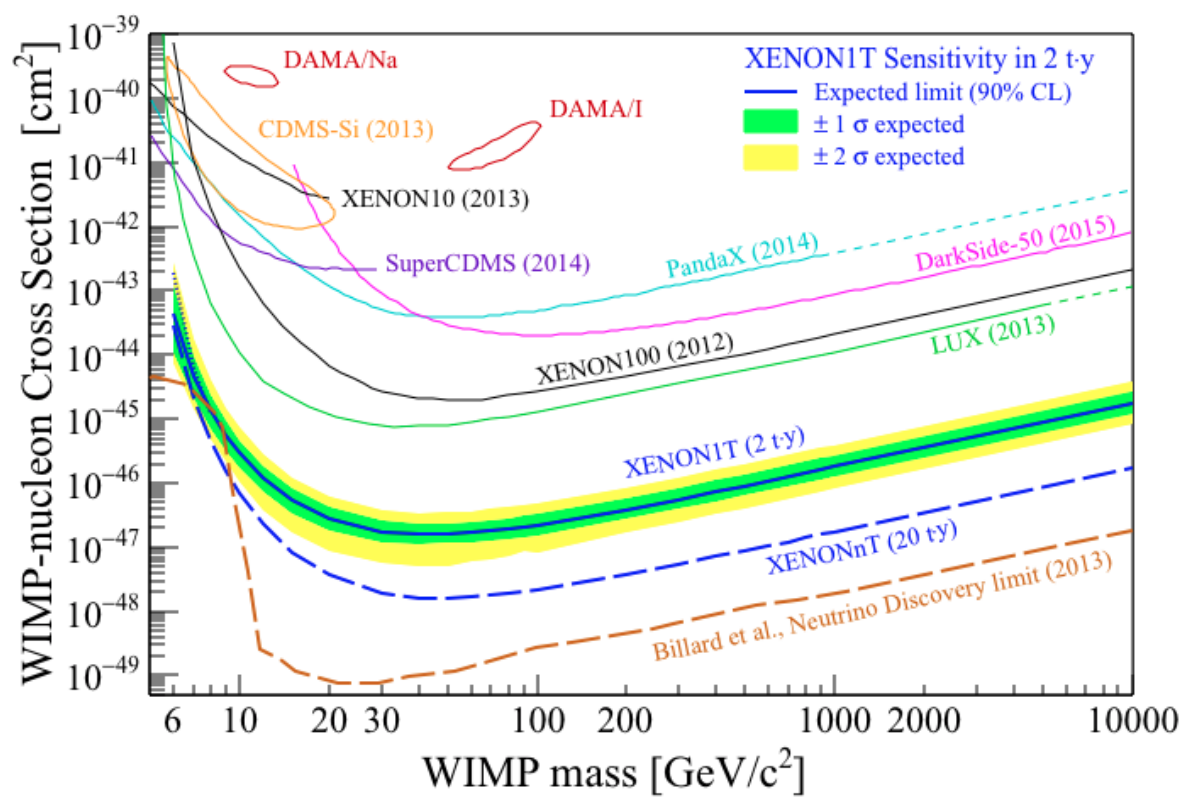

Figure 8: XENON1T sensitivity (90\% C.L.) to spin-independent WIMP-nucleon interaction [9] 


\section{Conclusions}

The XENON program aims at direct detection of Weakly Interacting Massive Particles with dual phase xenon time projection chambers located at the Laboratori Nazionali del Gran Sasso, in Italy. After the successful operation of XENON100 - for many years the world's most sensitive deep underground WIMP detector - the next generation detector XENON1T is ready to start data taking by the end of spring 2016. XENON1T will be the first experiment to use liquid xenon in a TPC at the ton scale. It is designed to achieve two orders of magnitude higher sensitivity than its predecessor.

\section{References}

[1] Planck Collaboration, P. A. R. Ade et al., "Planck 2015 results. XIII. Cosmological parameters," arXiv:1502.01589 [astro-ph.CO].

[2] D. N. Spergel, “The Motion of the Earth and the Detection of Wimps," Phys. Rev. D37 (1988) 1353.

[3] T. Marrodán Undagoitia and L. Rauch, "Dark matter direct-detection experiments," J. Phys. G43 no. 1, (2016) 013001, arXiv:1509.08767 [physics.ins-det] .

[4] XENON Collaboration, J. Angle et al., "First Results from the XENON10 Dark Matter Experiment at the Gran Sasso National Laboratory,” Phys. Rev. Lett. 100 (2008) 021303, arXiv: 0706.0039 [astro-ph].

[5] XENON10 Collaboration, J. Angle et al., "A search for light dark matter in XENON10 data," Phys. Rev. Lett. 107 (2011) 051301, arXiv:1104.3088 [astro-ph. CO] . [Erratum: Phys. Rev. Lett.110,249901(2013)].

[6] XENON100 Collaboration, E. Aprile et al., "The XENON100 Dark Matter Experiment," Astropart. Phys. 35 (2012) 573-590, arXiv:1107.2155 [astro-ph.IM] .

[7] XENON100 Collaboration, E. Aprile et al., "Dark Matter Results from 225 Live Days of XENON100 Data," Phys. Rev. Lett. 109 (2012) 181301, arXiv:1207.5988 [astro-ph.CO] .

[8] XENON1T Collaboration, E. Aprile, “The XENON1T Dark Matter Search Experiment," Springer Proc. Phys. 148 (2013) 93-96, arXiv:1206.6288 [astro-ph.IM] .

[9] XENON Collaboration, E. Aprile et al., "Physics reach of the XENON1T dark matter experiment," JCAP 1604 no. 04, (2016) 027, arXiv:1512.07501 [physics.ins-det] .

[10] XENON100 Collaboration, E. Aprile et al., "Limits on spin-dependent WIMP-nucleon cross sections from 225 live days of XENON100 data," Phys. Rev. Lett. 111 no. 2, (2013) 021301, arXiv:1301.6620 [astro-ph.CO].

[11] XENON100 Collaboration, E. Aprile et al., "First Axion Results from the XENON100 Experiment," Phys. Rev. D90 no. 6, (2014) 062009, arXiv:1404.1455 [astro-ph. CO] .

[12] R. Bernabei et al., "Final model independent result of DAMA/LIBRA-phase1," Eur. Phys. J. C73 (2013) 2648, arXiv:1308.5109 [astro-ph.GA] .

[13] XENON100 Collaboration, E. Aprile et al., "Exclusion of Leptophilic Dark Matter Models using XENON100 Electronic Recoil Data,” Science 349 no. 6250, (2015) 851-854, arXiv:1507.07747 [astro-ph.CO]. 
[14] XENON100 Collaboration, E. Aprile et al., "Search for Event Rate Modulation in XENON100 Electronic Recoil Data," Phys. Rev. Lett. 115 no. 9, (2015) 091302, arXiv: 1507.07748 [astro-ph. CO].

[15] XENON Collaboration, E. Aprile et al., "Lowering the radioactivity of the photomultiplier tubes for the XENON1T dark matter experiment," Eur. Phys. J. C75 no. 11, (2015) 546, arXiv:1503.07698 [astro-ph.IM].

[16] K. Lung et al., "Characterization of the Hamamatsu R11410-10 3-Inch Photomultiplier Tube for Liquid Xenon Dark Matter Direct Detection Experiments,” Nucl. Instrum. Meth. A696 (2012) 32-39, arXiv:1202.2628 [physics.ins-det].

[17] L. Baudis, A. Behrens, A. Ferella, A. Kish, T. Marrodan Undagoitia, D. Mayani, and M. Schumann, "Performance of the Hamamatsu R11410 Photomultiplier Tube in cryogenic Xenon Environments," JINST 8 (2013) P04026, arXiv:1303.0226 [astro-ph. IM] .

[18] XENON Collaboration, A. Lyashenko, "Progress in characterization of the Photomultiplier Tubes for XENON1T Dark Matter Experiment,” in 2014 IEEE Nuclear Science Symposium and Medical Imaging Conference and 21st Symposium on Room-Temperature Semiconductor X-ray and Gamma-ray Detectors (NSS/MIC 2014 / RTSD 2014) Seattle, WA, USA, November 8-15, 2014. 2015. arXiv:1502.01000 [astro-ph.IM]. http://inspirehep.net/record/1342781/files/arXiv:1502.01000.pdf.

[19] A. Lyashenko, T. Nguyen, A. Snyder, H. Wang, and K. Arisaka, "Measurement of the absolute Quantum Efficiency of Hamamatsu model R11410-10 photomultiplier tubes at low temperatures down to liquid xenon boiling point," JINST 9 no. 11, (2014) P11021, arXiv: 1410.3890 [astro-ph. IM] .

[20] XENON1T Collaboration, E. Aprile et al., "Conceptual design and simulation of a water Cherenkov muon veto for the XENON1T experiment," JINST 9 (2014) 11006, arXiv:1406.2374 [astro-ph. IM] .

[21] E. Aprile et al., "Performance of a cryogenic system prototype for the XENON1T Detector," JINST 7 (2012) P10001, arXiv:1208.2001 [physics.ins-det].

[22] S. Rosendahl, E. Brown, I. Cristescu, A. Fieguth, C. Huhmann, M. Murra, and C. Weinheimer, “A cryogenic distillation column for the XENON1T experiment,” J. Phys. Conf. Ser. 564 no. 1, (2014) 012006.

[23] E. Aprile, T. Yoon, A. Loose, L. W. Goetzke, and T. Zelevinsky, “An atom trap trace analysis system for measuring krypton contamination in xenon dark matter detectors," Rev. Sci. Instrum. 84 (2013) 093105, arXiv:1305.6510 [physics.atom-ph].

[24] S. Lindemann and H. Simgen, "Krypton assay in xenon at the ppq level using a gas chromatographic system and mass spectrometer," Eur. Phys. J. C74 (2014) 2746, arXiv:1308. 4806 [physics.ins-det]. 